\title{
LAS ESTACIONES EN LA LÍNEA DE ALTA VELOCIDAD ESPAÑOLA
}

(TRAIN STATIONS ON THE SPANISH HIGH SPEED ROUTE)

Julio Barcones Mingueza, Ingeniero de Caminos

Jefe de Infraestructura de AVE

Alfonso González, Ingeniero Industrial

Gerente Obras Córdoba y Sevilla AVE

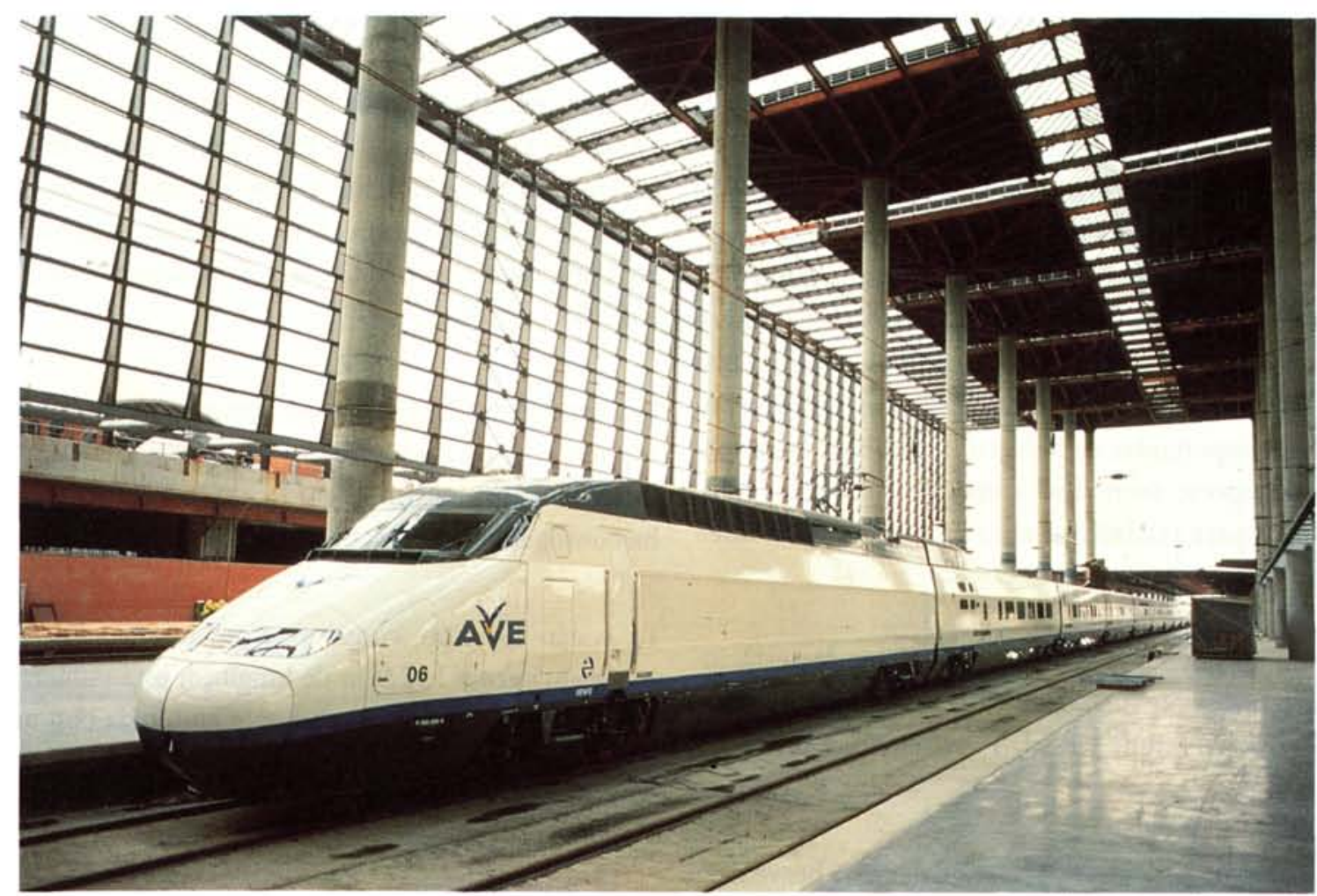

Andén en la anueva" Estación de Atocha-Madrid.

Foto: PATIER

RESUMEN

Con una breve descripción de las estaciones de MadridAtocha, Ciudad Real, Puertollano, Córdoba y Sevilla se pretende proporcionar al lector una visión de los aspectos más relevantes de estos centros de conexión del viajero con los trenes de AVE.

\section{SUMMARY}

With a brief description of the stations in Madrid (Atocha), Ciudad Real, Puertollano, Cordoba and Seville, the article attempts to provide the reader with a vision of the most relevant aspects of these four centers of connection for the traveler on the high speed route. 


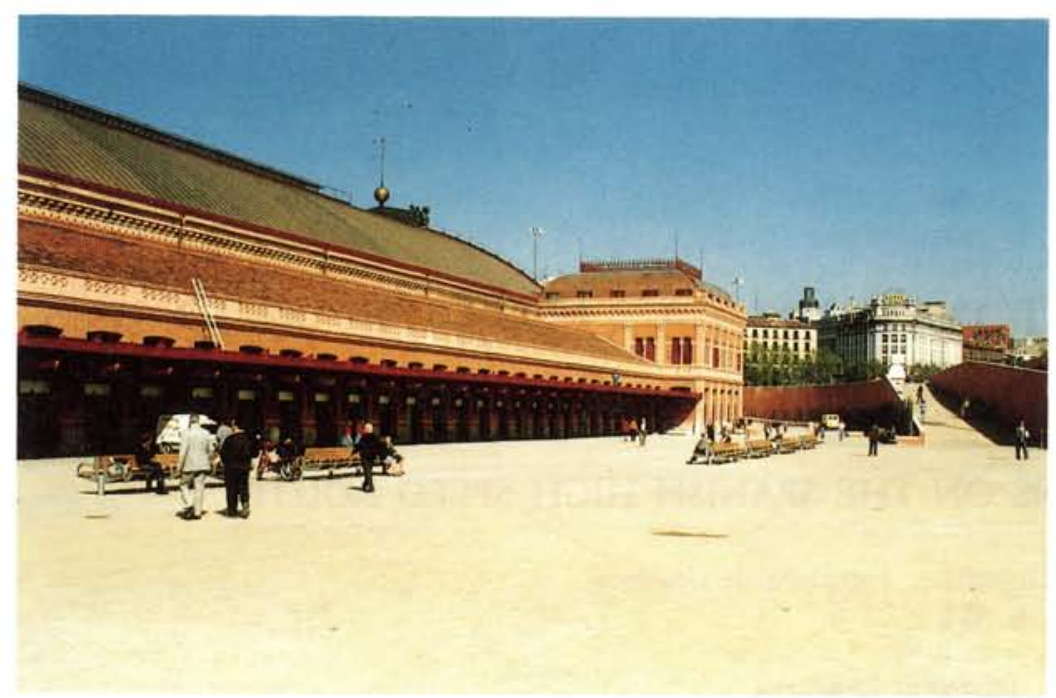

Acceso a la "vieja» Estación de Atocha-Madrid.

\section{INTRODUCCIÓN}

Denominamos a una línea ferroviaria, de Alta Velocidad, cuando permite que los vehículos que por ella circulan desarrollen velocidades superiores a los $200 \mathrm{~km} / \mathrm{h}$. Es indudable, que para conseguir una velocidad comercial alta (171 km/h en Madrid-Sevilla) es muy importante lo indicado anteriormente, pero también el número de paradas comerciales que es preciso efectuar, razón por la cual estas paradas deben realizarse únicamente en núcleos importantes de población, y utilizar otros medios de transporte alternativo: trenes de cercancías, autobuses, etc., para trasladarse a las estaciones de la línea de Alta Velocidad.

Teniendo en cuenta lo anterior, cinco son las estaciones que actualmente funcionan en la línea Madrid-Sevilla: Madrid-Atocha, Ciudad Real, Puertollano, Córdoba y Sevilla.

Con el presente artículo se pretende dar una breve descripción de cada una de ellas, que permita al lector una somera visualización de lo que se encontrará cuando las visite.

\section{MADRID-ATOCHA}

Situada en el origen de la línea, ocupa los terrenos de la antigua estación de Atocha cumpliendo uno de los objetivos de las estaciones, el de situarse lo más cerca - consejo del centro de gravedad de la cior de Investigâciones Científicas
Es indudable que las necesidades a satisfacer por una estación actual, con vistas de futuro, no podían conseguirse sin una remodelación de la estación que se terminó de construir y se inauguró en 1892, según proyecto del arquitecto Alberto Palacios, y que fue adecuándose a las necesidades del servicio ferroviario hasta que se cerró en 1988 para construir la actual, inaugurada el 14 de abril de este año.

Era importante, teniendo en cuenta lo que para Madrid supone la «vieja» estación de Atocha, que en el proyecto de la nueva, se integrara la antigua. Esto es lo que ha conseguido el proyecto del arquitecto Rafael Moreno.

La «vieja» estación, constituida por una nave de 46,76 $\mathrm{m}$ de anchura y $152,9 \mathrm{~m}$ de longitud, está cubierta por una bóveda de cañón ligeramente apuntada con una altura de $27 \mathrm{~m}$. En la clave, un lucernario permite su ventilación e iluminación. Esta nave está precedida por un pabellón de entrada, encima del cual una gran cristalera le sirve de cierre.

Este cuerpo de entrada, de cubierta plana, va flanqueado por dos torres, de las que arrancan los cuerpos laterales.

El conjunto arquitectónico descrito albergaba las dependencias necesarias para dar servicio al tráfico de trenes que utilizaban las seis vías que, agrupadas de dos en dos, estaban bajo la marquesina y eran servidas longitudinalmente por cuatro andenes.

http://informesdelaconstruccion.revistas.csic.es 
Es indudable que este espacio, que durante 100 años hizo posible la explotación ferroviaria, no permitía acoger las vías y andenes necesarios para el tráfico actual y las previsiones futuras. Por esta razón, la «vieja» estación pasa a ser el gran vestíbulo de la nueva estación de Atocha. Aloja en sus pabellones laterales y torres las dependencias necesarias para el buen funcionamiento de la estación, así como el comercio que los usuarios demandan hoy como un servicio ferroviario más. Para conseguir lo indicado ha sido preciso demoler tabiquería, forjados y falsos techos de escayola, que desvirtuaban las condiciones especiales primitivas; se han levantado los tendidos aéreos colocados a la fachada; se han demolido toperas y levantado las vías, y se han rehabilitado o restituido todas las piezas arquitectónicas que estaban deterioradas, de forma que la estación pudiera recobrar su antiguo esplendor.

Mención esencial en esta rehabilitación merecen los trabajos de la fachada de vidrio, la cubierta de la nave central y el tratamiento del material cerámico.

La fachada de vidrio, denominada en el proyecto de Alberto Palacios «videau vitree», presentaba un acentuado pandeo de los perfiles principales, por lo que ha sido precisa una sustitución importante de las mismas, así como una complementaria de la perfilería de orden secundario y la de recibido de los vidrios.

La cubierta de la nave central ha sido levantada en su totalidad, sustituyendo el fibrocemento por láminas de cobre.

Respecto al material cerámico, dieciséis han sido los tipos de piezas que se han rehabilitado o sustituido, restituyendo los muros a su estado ornamental inicial.

Recuperada la situación primitiva del edificio, se le han agregado elementos que complementan su funcionalidad y su belleza.

Entre los primeros, un forjado a la cota $622,6 \mathrm{~m}$ en las naves laterales y pabellón central permite la ubicación de espacios para usos comerciales intercomunicados por pasillo perimetral. En la cota 619,2 m (nivel de la nave), y adosada al núcleo central, se cimentó una losa situada en la cota 622,6 que comunica con el pasillo perimetral indicado anteriormente, y permite nuevos espacios comerciales en la cota 619,2 metros.
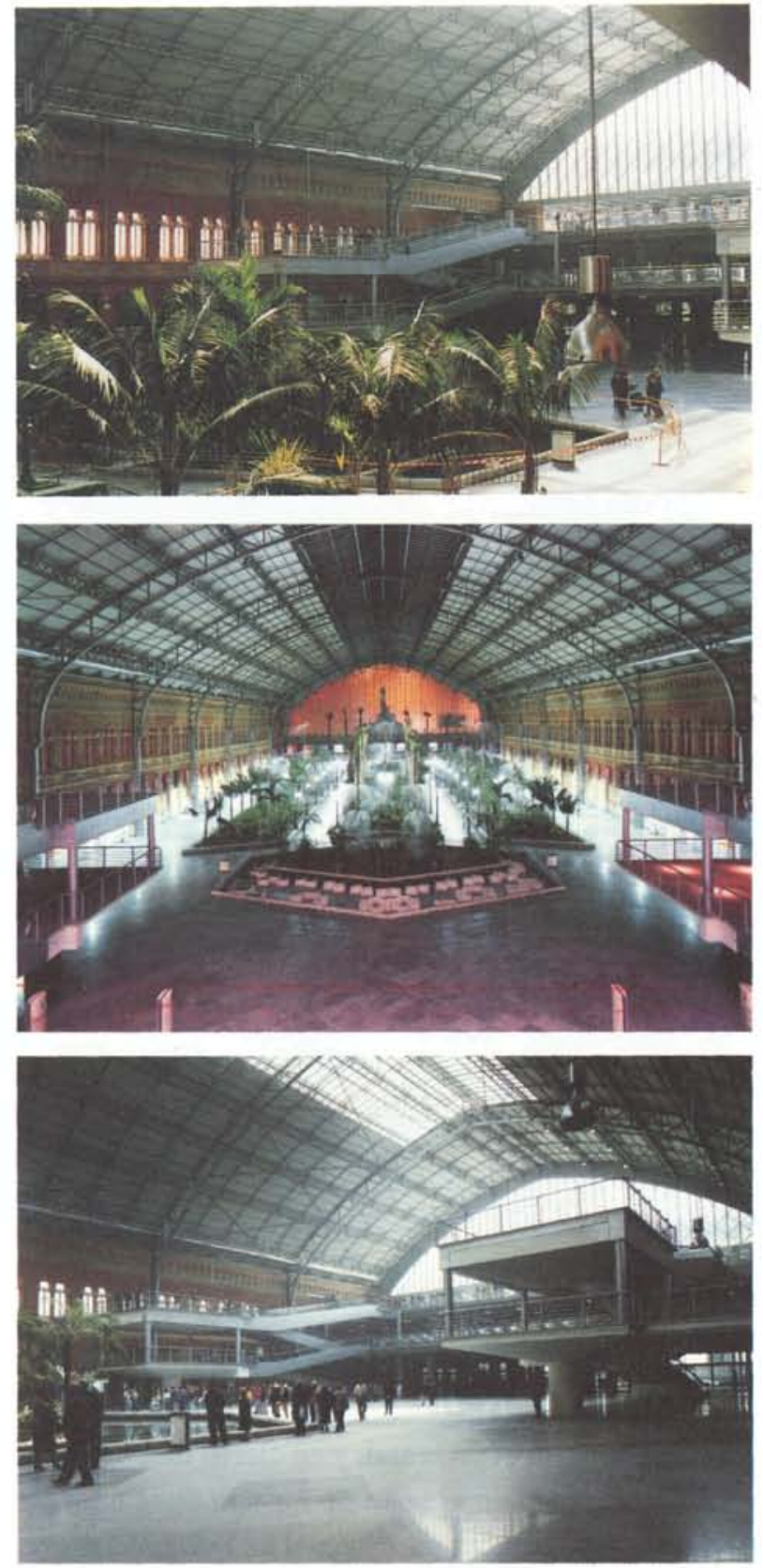

Nave central.

Foto: IILLAR, S.1

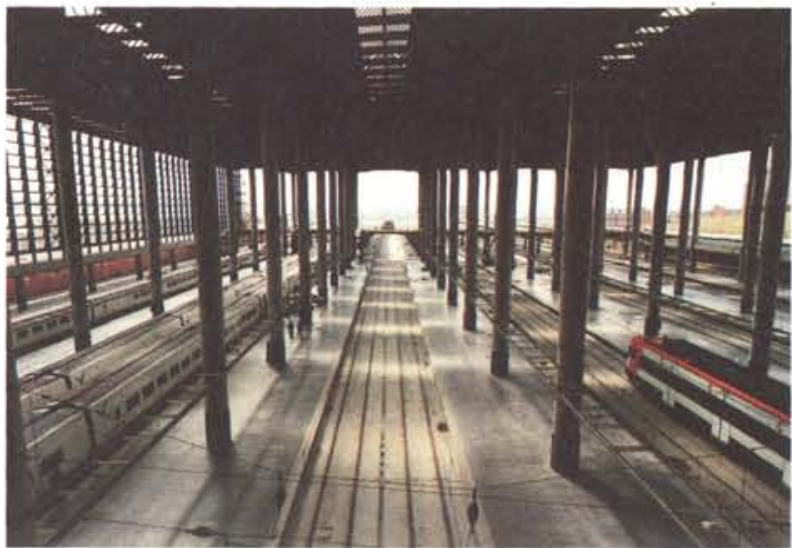

Andenes.

Foto: VILLAR, S.L.

http://informesdelaconstruccion.revistas.csic.es 
Informes de la Construcción, Vol. 44 n. ${ }^{\circ}$ 420, julio/agosto, 1992

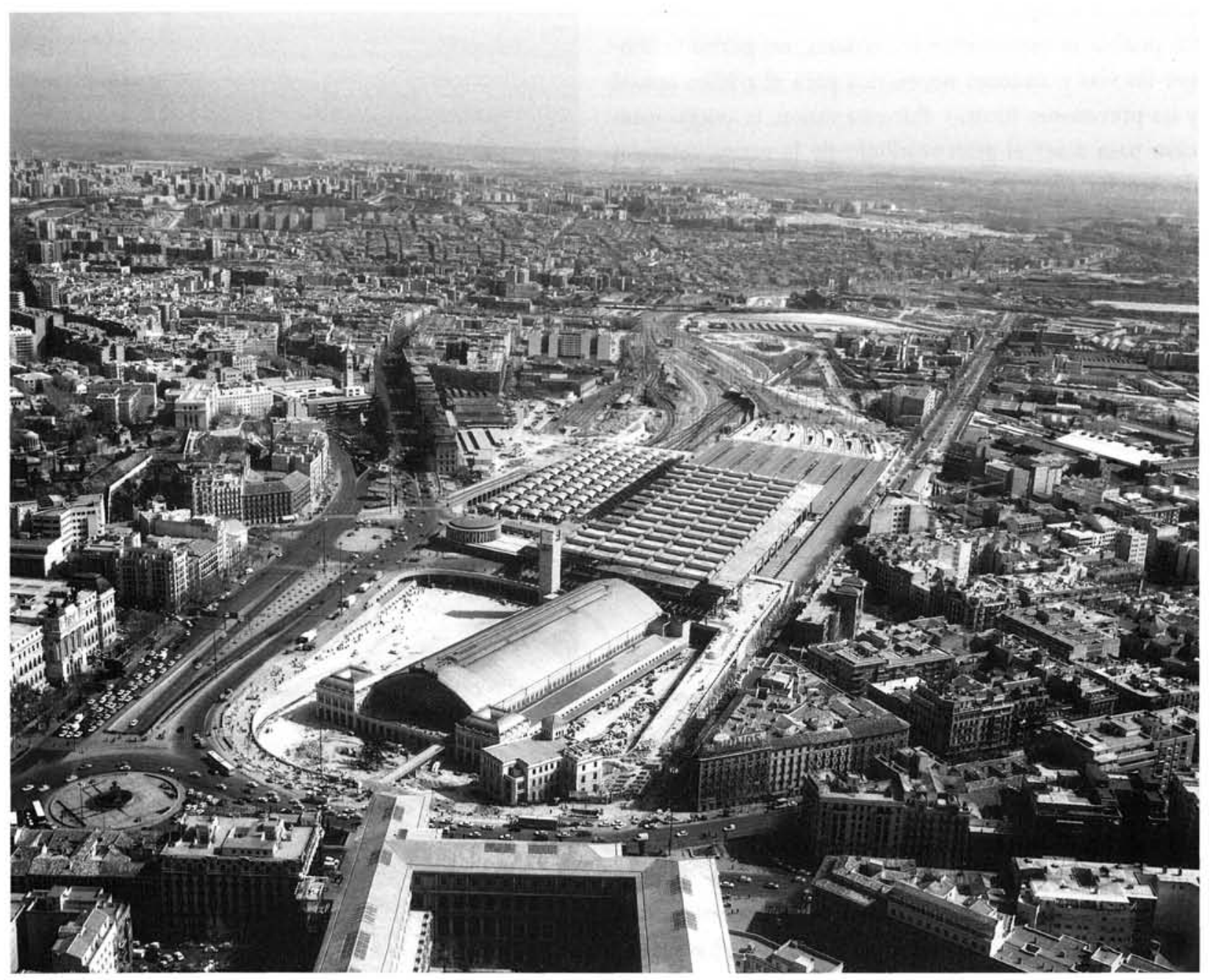

Vista general del complejo ferroviario de la Estación de Atocha.

Foto: ALTAIR PHOTO, S.A.

Como elemento ornamental, y en cierto modo exótico, se ha proyectado en el resto de espacio de la nave central un jardín tropical, para lo cual ha sido preciso crear un microclima especial basado en el llamado «efecto invernadero", conseguido con el cerramiento superior en cristal, y empleando plantas tropicales y subtropicales que se aclimataran al recinto como consecuencia del empleo de diversos mecanismos de climatización y control medioambiental regulados automáticamente, y que reproducirán lo más fielmente posible los climas de donde son originarias estas plantas.

En este jardín podrá encontrar el visitante las más variadas especies propias de una selva tropical, la amazónica.

Como nexo de unión entre las instalaciones anteriormente descritas, y las vías y andenes donde el viajero accederá (c) Consejo Superior de Investigaciones Científicas cotas: $616,2 \mathrm{~m}$, Licencia Creative Commons 3.0 España (by-nc)
624,5 y $628,7 \mathrm{~m}$, que tienen como misión fundamental la intercomunicación en vertical con otros modos de transporte: trenes de cercanías, metro, autobuses, taxis.

La losa en la cota 619,2 se corresponde con el nivel de pavimentación de la marquesina, permitiendo el acceso directo a andenes, el peatonal desde la plaza de la estación, así como el servicio público de taxis.

La cota 624,5 se proyecta como un gran hall de espera que permite el acceso directo a una entreplanta de cercanías, así como al aparcamiento público ubicado sobre las vías de cercanías.

La cota $628,7 \mathrm{~m}$ permite el acceso a la estación de los vehículos, estando a nivel de la glorieta a la que confluyen las calles Infanta Isabel, Alfonso XII y Avenida Ciudad de Barcelona. 
Para establecer las comunicaciones entre estos tres niveles se han diseñado 3 sistemas diferenciados e integrados en las losas indicadas: rampas mecánicas con rellanos intermedios, en las cotas 622,6 y 626 , situadas en el lado de la gran marquesina y próximas a las naves laterales; escaleras mecánicas que comunican con cotas 619,2 y 624,5 situadas por el lado toperas y centradas respecto a las vías; dos grupos de ascensores hidráulicos situados en los extremos norte y sur de las zonas y que comunican los tres niveles; y escaleras fijas de comunicación con las tres plantas que arrancan de la plaza de la estación y del patio de Méndez Álvaro.

En total son 15 el número de vías que dan servicio a la estación, de las que 7 son de ancho UIC (1.435 mm), y 8 de ancho RENFE (1.668 mm). La longitud útil es superior a los $500 \mathrm{~m}$, excepto en la más próxima a cercanías que tiene $476 \mathrm{~m}$. Para el servicio de estas vías existen ocho andenes de $10 \mathrm{~m}$ de anchura, excepto el más próximo a la calle Méndez Álvaro que tiene 9,17 $\mathrm{m}$. Las vías se han proyectado con carril soldado en toda su longitud y apoyado en lecho elástico "corkelat» sobre losa de hormigón. Los andenes se han diseñado con dos muretes de hormigón, que se rellenan entre ellos con suelo seleccionado y solera de hormigón apoyada en los muretes, y con un pequeño vuelo sobre el que se coloca una pieza de granito.

Las marquesinas çubren en $500 \mathrm{~m}$ todos los andenes y vías. Los primeros $250 \mathrm{~m}$ se solucionan con la llamada "sala hipóstila» en la que la cubierta metálica se sustenta sobre pilares cilíndricos de $22,5 \mathrm{~m}$ de altura y 1,2 $\mathrm{m}$ de diámetro, formando una retícula esviada de 16,6 $\mathrm{m}$ por 17,66 $\mathrm{m}$. Las uniones de esta retícula metálica crean pasillos centrales abiertos en las dos direcciones principales paralelas al eje de la estación de cercanías, y a la línea de fachada de la estación de largo recorrido.

El resto de las marquesinas son bajas. Su cubierta está sustentada sobre pilares cilíndricos de un metro de diámetro que se sitúan según la malla de 16,0 por 17,66 , y no cubren la totalidad de la superficie como en la «sala hipóstila», sino únicamente los andenes y parte de las vías contiguas.

En la zona próxima a cercanías, un muro cortina de 16,10 de altura cierra el espacio que quedó verticalmente entre la "sala hipóstila» y las marquesinas bajas.
En el punto de encuentro de la sala hipóstila y las marquesinas bajas se ha construido, transversalmente a las vías, una pasarela a la cota 624 , que comunica directamente con el aparcamiento existente sobre las vías de cercanías, y al mismo tiempo permite el paso entre andenes, para lo cual existe en cada andén unas rampas fijas.

Para el acceso de vehículos públicos en el patio de Méndez Álvaro, desde la losa de la cota 628,7 , se ha construido una rampa de $2,5+9+2,5 \mathrm{~m}$ de anchura con una pendiente del $5 \%$ que salva el desnivel hasta la cota 619.

Como complemento a todo lo anterior está la gran plaza peatonal, en el lado norte del edificio histórico, situado por debajo del nivel de la Glorieta de Carlos V, de la que se separa perimetralmente mediante un muro de hormigón armado, trasdosado con fábrica de ladrillo visto, rematado con imposta de ladrillo y que va desde el borde del túnel de penetración de Móstoles, hasta la conexión con el acceso a cercanías.

La plaza peatonal está pavimentada mediante adoquinado de hormigón apoyado sobre lecho de arena en losa de hormigón armado de $20 \mathrm{~cm}$ de espesor, creándose zonas ajardinas en las proximidades de la pasarela que, partiendo de la Glorieta de Carlos V, accede al edificio en la zona de «la cortina vidriada» a la cota 626,7 .

\section{CIUDAD REAL}

La nueva estación (la segunda de la línea) está situada al este de la ciudad, junto al acceso de la CN-430, que une esta capital con Puerto Lápice y, por tanto, con la autovía de Andalucía.

A la nueva estación de Ciudad Real accederán tanto los trenes AVE, que procedan de la relación Madrid-Sevilla, como los convencionales de la de Madrid-Badajoz. Una de sus características fundamentales es, por tanto, la de permitir una explotación conjunta, aunque diferenciada, de los dos tipos de ferrocarril. Por ello, en su diseño se han tenido en cuenta las necesidades y equipamientos que cada uno de dichos ferrocarriles demanda.

La estación en sí se divide en infraestructura y playa de vías, plaza de acceso, edificio y aparcamientos y edificios anexos. La infraestructura se define por un terra- 


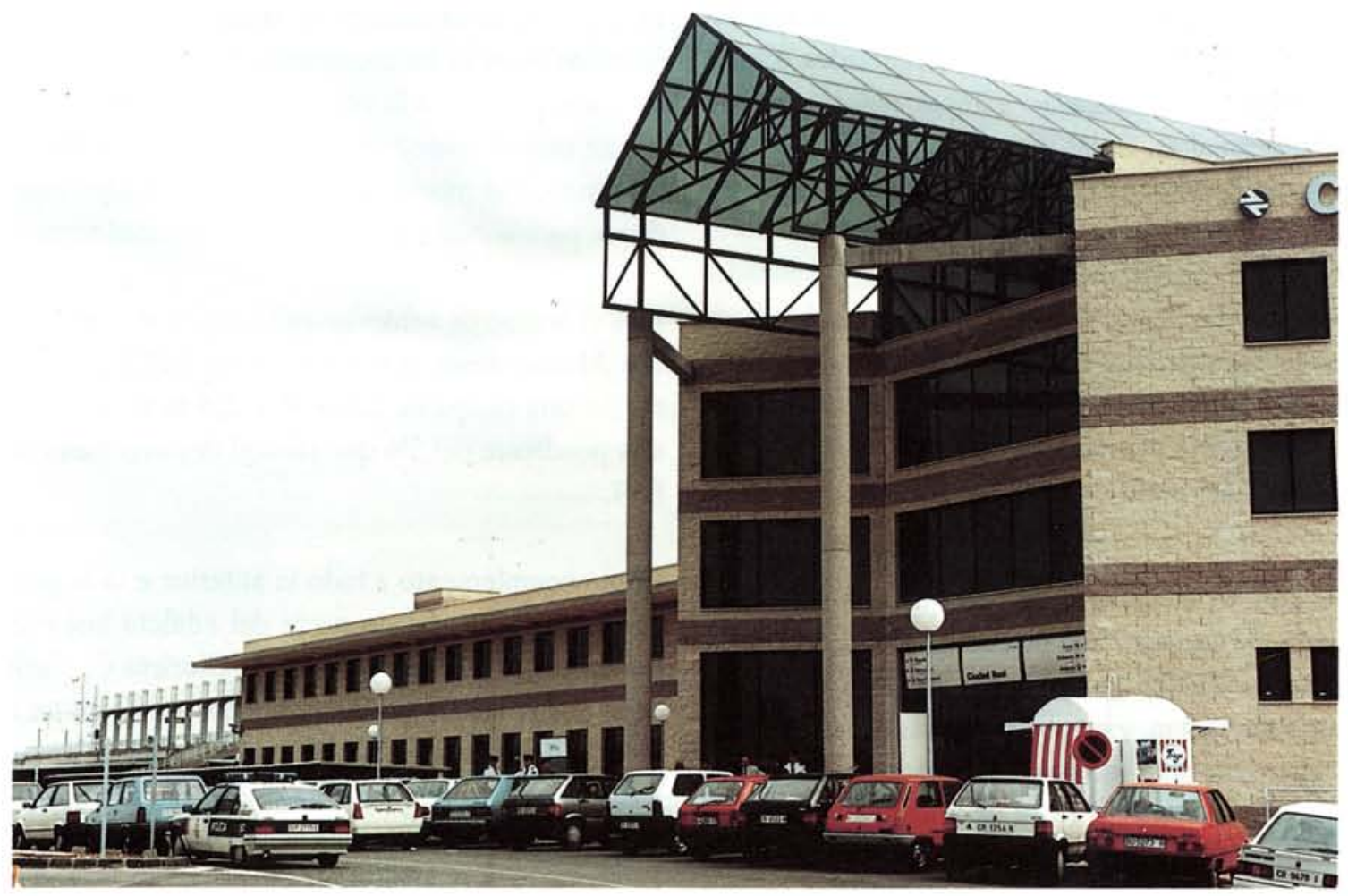

Estación de Ciudad Real.

plén de $6 \mathrm{~m}$ de altura, con una longitud de $1 \mathrm{~km}$ y un ancho de $60 \mathrm{~m}$ en la zona de andenes. Sobre él se asienta un haz de vías con ancho 1,435 (AVE) y otro con ancho 1,668 (RENFE). El primero consta de dos vías generales y dos de apartado, a las que se accede mediante desvíos de tangente 0,071 con corazón móvil. Asimismo, se disponen culatones de maniobra en cada una de estas vías de apartado, según el sentido de la marcha. A ambos lados de la estación, y con el fin de poder cambiar de vía los trenes de Alta Velocidad, se disponen escapes formados por desvíos de tangente 0,026 enlazados.

El segundo haz de vías mantiene las dos generales (a Badajoz o a Madrid), con una geometría idéntica a los de AVE, y una distancia de $9 \mathrm{~m}$ entre los ejes de los más próximos y de ancho diferente. Estas vías se ramifican en otra de circulación y un culatón de maniobras; todas ellas terminan con toperas, ya que esta parte de estación funcionará como si fuera de término. Los desvíos utilizados son del tipo B y las traviesas bivalentes.

Existen tres andenes: el principal, junto al edificio de viajeros de $450 \mathrm{~m}$ de longitud, con un ancho de $6 \mathrm{~m}$;

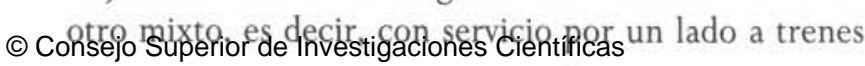
Licencia Creative Commons 3.0 España (by-nc) de Alta Velocidad y por otro a trenes convencionales también de $450 \mathrm{~m}$ de largo por $10 \mathrm{~m}$ de ancho; y un tercero para trenes convencionales de $250 \mathrm{~m}$. Los dos primeros van cubiertos por marquesinas en un 60 por 100 de su longitud, y el tercero en el 100 por 100 .

La plaza de acceso corresponde a una extensa zona urbanizada delante de la fachada principal del edificio de viajeros, que enlaza con una gran avenida prevista en la modificación del Plan General de Ordenación como acceso principal a estas instalaciones ferroviarias.

El edificio principal consta de un gran vestíbulo central triangular y dos alas laterales rectangulares. El vestíbulo tiene una superficie de $970 \mathrm{~m}^{2}$ con un 50 por 100 totalmente diáfano en toda su altura, que permite la iluminación natural a través de una cubierta traslúcida. En este vestíbulo se encuentran agrupadas todas las dependencias de atención al viajero: la facturación y recogida de equipajes a la izquierda, el acceso al andén principal mediante rampas mecánicas y cafetería a la derecha, así como taquillas, información general y turística en el centro. También en este hall se encuentra la sala Rail-Club y una pequeña zona destinada a locales comerciales. http://informesdelaconstruccion.revistas.csic.es 


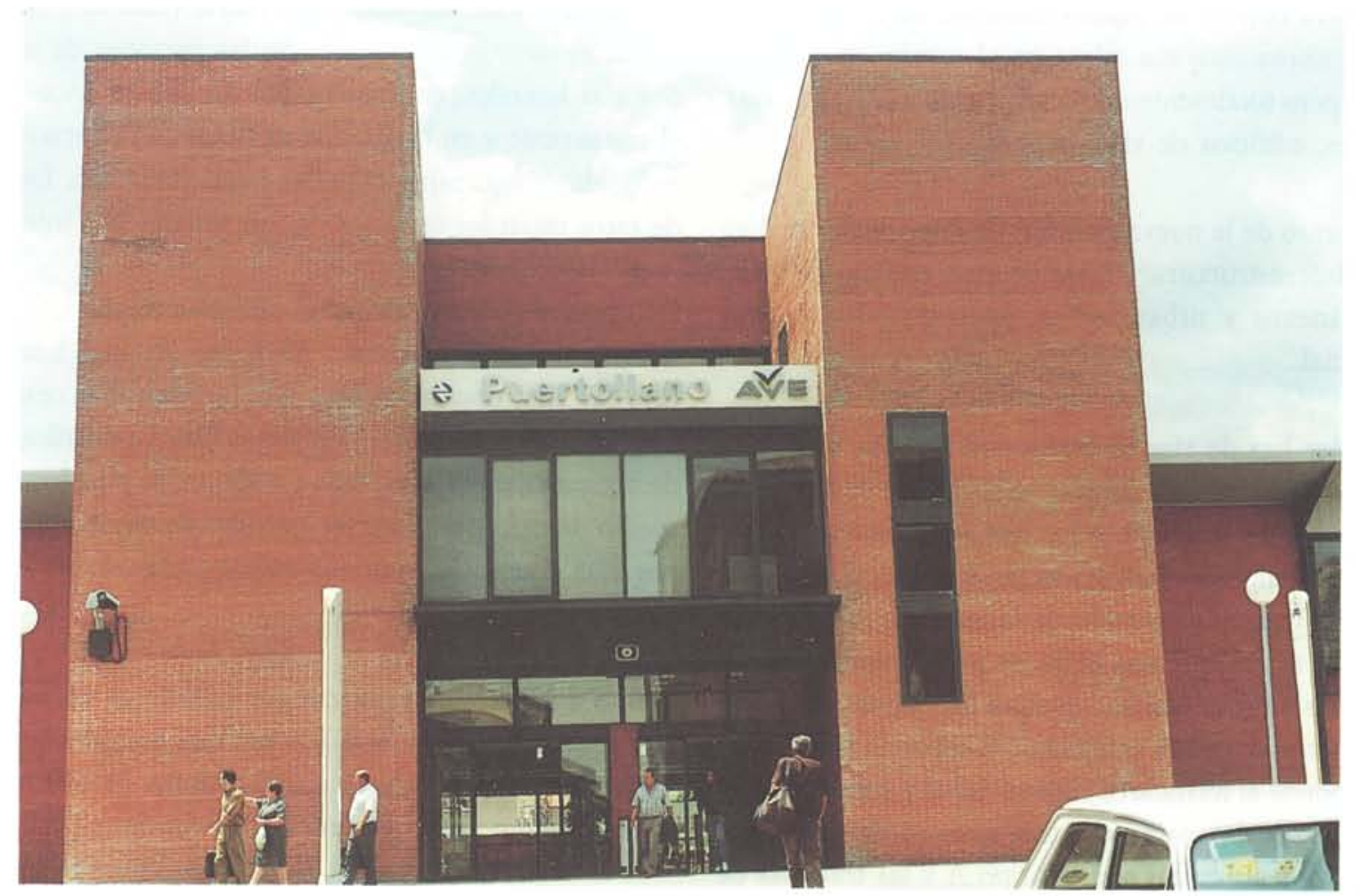

Estación de Puertallano.

Con esta distribución se simplifican las circulaciones peatonales, de manera que el usuario pueda localizar desde el acceso principal todas las dependencias y servicios que se ponen a su disposición. Las plantas primera y segunda se utilizan pará uso interno con una superficie de $500 \mathrm{~m}^{2}$ por planta. Anejo a este hall y a la izquierda se levanta un ala de planta rectangular en dos alturas $(2.160$ $\mathrm{m}^{2}$ ), en el que se encuentran una zona de servicios internos en planta baja y oficinas en la primera.

Con el fin de distribuir los equipajes a cada uno de los andenes se ha previsto un sistema de rampas fijas para la circulación de las carretillas eléctricas, que unen cada uno de dichos andenes con la zona de recogida de facturación. La longitud es de 150 m con un tratamiento especial antideslizante en la solera. Parte de esta longitud va volada sobre una estructura y parte bajo las vías. A la derecha del hall y sobre la cafetería, en planta primera se sitúa un restaurante de $400 \mathrm{~m}^{2}$ con acceso directo al andén principal.

El aparcamiento se halla en el lado opuesto al edificio, es decir, al otro lado del terraplén base de la playa de vías. En esta zona existe una gran superficie para zona (c) Consejo Superior de Investigaciones Científicas Licencia Creative Commons 3.0 España (by-nc) de aparcamiento $\left(3.500 \mathrm{~m}^{2}\right)$, zonas verdes $\left(2.500 \mathrm{~m}^{2}\right)$, así como el edificio de Paquete-Expres y el de Correos, ambos de planta rectangular $\left(420 \mathrm{~m}^{2}\right.$, cada uno), y el edificio técnico soporte del enclavamiento electrónico de la estación AVE $\left(500 \mathrm{~m}^{2}\right)$.

La comunicación entre la plaza de acceso, el edificio y esta zona se consigue mediante un paso inferior de $60 \mathrm{~m}$ de longitud y $30 \mathrm{~m}$ de ancho, que recoge tanto las circulaciones peatonales como el tráfico viario, logrando con ello una mayor iluminación del conjunto. Para separar los dos ambientes se coloca un muro cortina, lo que permite la continuidad espacial. La zona peatonal abarca $1 / 3$ del total del paso y en ella existe una serie de locales comerciales a lo largo de toda su longitud. Además, desde ésta y mediante rampas mecánicas, escaleras fijas y ascensores, se puede acceder a cada uno de los andenes. Esta zona peatonal comunica con el ala derecha del edificio.

\section{PUERTOLLANO}

El paso de la línea de Alta Velocidad Madrid-Sevilla por Puertollano obliga a conjuntar las instalaciones de este 
ferrocarril con las de Madrid-Badajoz. Para ello se ubica una nueva estación mixta en el mismo sitio que el actual, pero totalmente nueva en cuanto a playas de vías, andenes, edificios de viajeros, etc.

El conjunto de la nueva estación de Puertollano se divide en Infraestructura y Playa de vías, edificio de viajeros y anexos y urbanización, aparcamientos y zona comercial.

El primer haz de vías de ferrocarril de Alta Velocidad es el más próximo al edificio y se compone de dos generales y dos de apartado, a los que se accede mediante desvío de tangentes 0,071 y se les dota de culatones de maniobra, según el sentido de la marcha. A ambos lados de la estación, y con el fin de poder cambiar de vía los trenes AVE, se instalan escapes formados por desvío de tangente de 0,085 enlazados. El segundo haz de vías corresponde al ferrocarril convencional y consta de uno general, tres de apartado y tres mangos de maniobra. Los desvíos utilizados son del tipo A y las traviesas de madera. El obligado trazado en curva de radio reducido de estas vías impone una velocidad máxima de paso inferior a los $200 \mathrm{~km} / \mathrm{h}$.

Existen tres andenes: El principal se sitúa en el edificio de viajeros, de $400 \mathrm{~m}$ de longitud; otro mixto, es decir, que da servicio tanto a los trenes de Alta Velocidad como a los convencionales, de $400 \mathrm{~m}$ de longitud; y un tercero para el ferrocarril convencional, de $295 \mathrm{~m}$ de longitud. Todos estos andenes van cubiertos por marquesinas.

El complejo de la estación se sitúa en el cruce de la calle Ancha con la calle del Muelle. Esta ubicación tiene conexión inmediata con el centro de la ciudad y simultáneamente permite una salida rápida hacia el norte bordeando las instalaciones ferroviarias. Según el planteamiento Municipal, el entorno de la estación posee el mayor índice de población residente potencial, siendo éste uno de los motivos que justifica el mantenimiento de la ubicación de estas instalaciones.

El edificio principal de la estación se desarrolla a partir de los dos prismas que limitan lateralmente el vestíbulo y cuyo eje coincide con el de la calle del Muelle, conectando la zona ferroviaria con el centro de la ciudad como ya se ha comentado. Desde estos prismas se extienden, a derecha e izquierda, las áreas públicas y restringidas de todo el edificio.
El edificio tiene un vestíbulo central $\left(220 \mathrm{~m}^{2}\right)$, desde donde el usuario distingue todas las opciones de uso y dos alas laterales, de manera que en una se encuentra el restaurante y en la otra los servicios de facturación y recogida de equipajes, taquillas y sala Rail-Club. Detrás de éstos están los servicios de funcionamiento interno.

El acceso al andén principal es directo a través del vestíbulo, y al resto de andenes mediante un paso inferior bajo las vías, con ascensores que facilitan la accesibilidad de los minusválidos, y escaleras fijas. La distribución de equipajes, entre el edificio y andenes, se establece por medio de otro paso inferior que accede mediante rampas fijas con suelo antideslizante a cada uno de estos andenes.

Frente a la fachada principal del edificio se encuentra una zona urbanizada de $8.706 \mathrm{~m}^{2}$, que comprende una parte destinada a viales y aparcamientos $\left(4.400 \mathrm{~m}^{2}\right)$ y el resto como zonas y verdes y peatonales.

\section{CÓRDOBA}

Las necesidades de Córdoba de articular una estructura urbana, acorde con el desarrollo de la ciudad hacia su zona norte, se encuentran "estranguladas" por el actual trazado ferroviario. Para dar una solución, se propone el proceso de remodelación de toda su Red Arterial Ferroviaria (RAF) permitiendo, a la vez, la concentración de las mercancías en la Estación de Mercancías de $\mathrm{El}$ Higuerón y una estación única de viajeros, que albergue tanto al de largo recorrido como al de cercancías.

La futura estación, actualmente en ejecución, abarca cuatro zonas principales: plaza de la estación, edificios, marquesinas y patio de andenes.

La plaza de la estación será peatonal y estará situada sobre el haz de vías de salida (lado Madrid), a una altura de $6,70 \mathrm{~m}$ sobre la cota de andenes, limitada al norte por una avenida de nueva formación, al sur por un futuro edificio destinado a servicios comerciales, al este por el paseo peatonal construido sobre el túnel y al oeste por la fachada del gran vestíbulo de acceso a andenes.

El conjunto arquitectónico total consta de las edificaciones para servicios centrales, viajeros, servicios ferroviarios y Correos. 


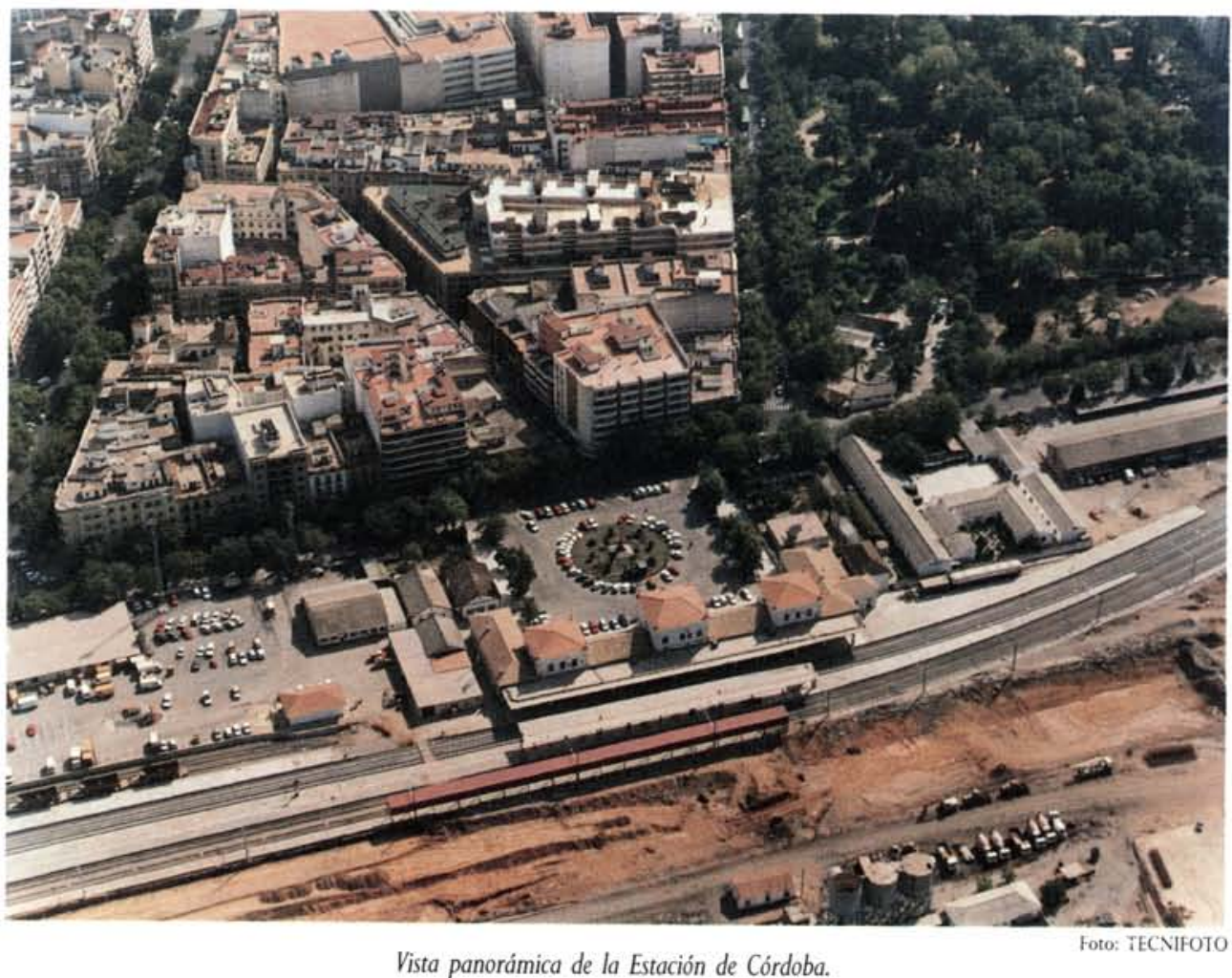

Vista panorámica de la Estación de Córdoba.

Las características técnicas de la estación de viajeros son las correspondientes a una solución de ferrocarril deprimido a una cota tal que la infraestructura se sitúe por encima del nivel freático, quedando soterrada en unos $3 \mathrm{~km}$ desde la zona de la estación de viajeros hasta la salida hacia Madrid. El edificio de la estación estará alineado con la Avenida de América, invadiendo el trazado de vías actuales.

Con el fin de liberar espacios para la ejecución de la estación indicada, ha sido preciso establecer una situación provisional, para lo que se ha tenido que realizar una variación de la traza actual de la vía, siguiendo en explotación la antigua estación con una mayor amplitud de espacio para el viajero.

Las actuaciones realizadas se pueden resumir de la siguiente forma:

\section{Para ancho RENFE}

- Construcción de una nueva vía desde Chinales hasta el P.S. del Brillante.

(c) Consejo Superior de Investigaciones Científicas Licencia Creative Commons 3.0 España (by-nc)
- Construcción de vías de cercanías junto al P.S. del Brillante, en el espacio ocupado por la antigua estación centro.

- Doble vía de salida hacia Sevilla desde el P.S. del Brillante al P.S. de la carretera de Palma del Río.

\section{Para Ancho Internacional}

- Dos vías paralelas a las de ancho RENFE, previéndose paradas momentáneas frente al edificio principal de viajeros.

- Como consecuencia de este montaje, se cortó la actual salida de ancho nacional hacia Málaga, siendo necesaria la realización de una nueva vía por CórdobaMercancías para que usando el salto del carnero existente sobre las vías de Sevilla, evite el cruce a nivel.

En la actualidad, una vez creadas las situaciones provisionales de ancho RENFE y Ancho Internacional, y en funcionamiento la estación de Córdoba-Mercancías, se están ejecutando las obras del soterramiento, las cuales han sufrido cierto retraso por la aparición de restos ar- 


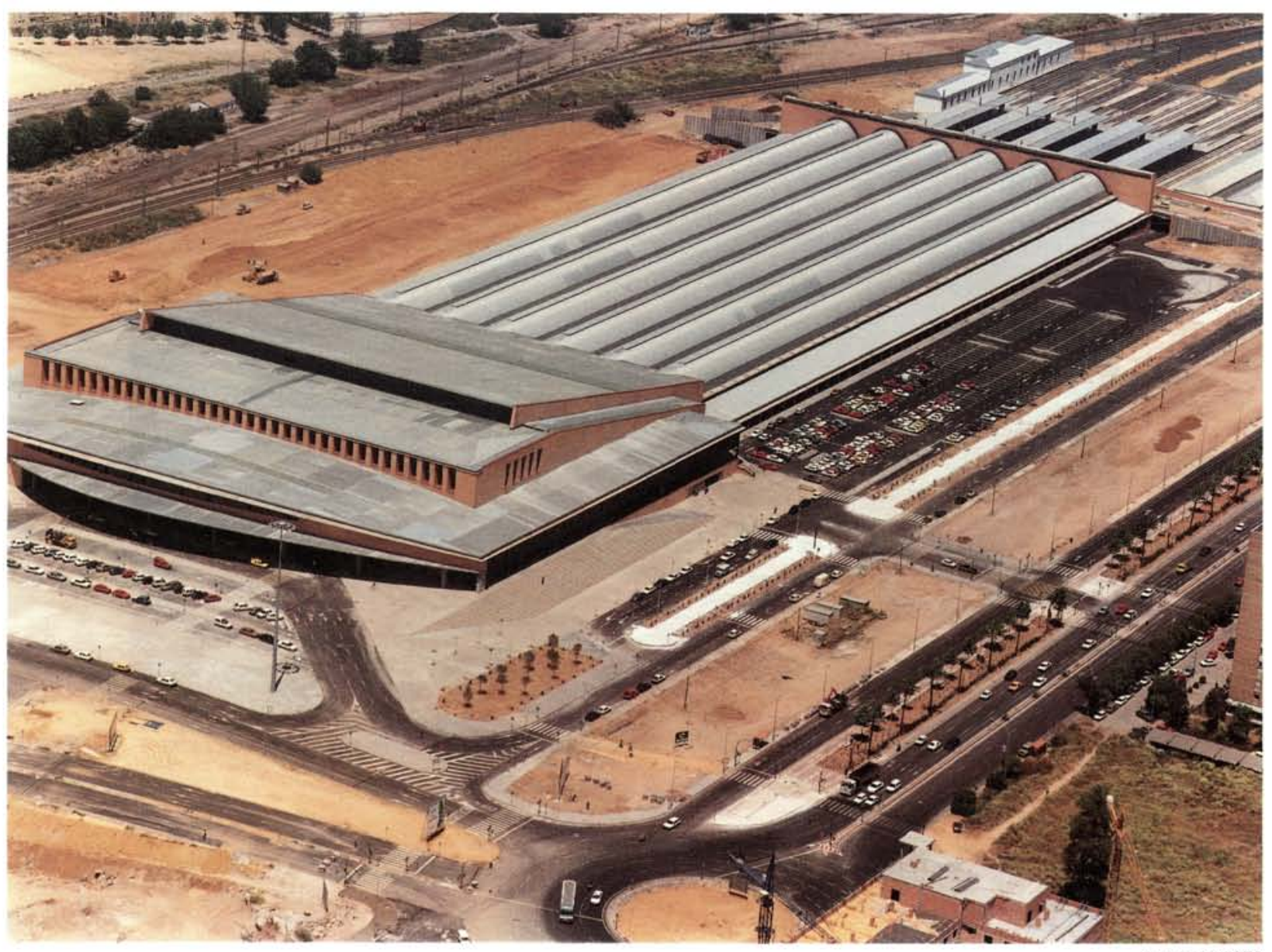

Vista panorámica de la Estación de Santa Justa-Sevilla. queológicos en la zona prevista para el emplazamiento del futuro edificio de viajeros.

La aparición de dichos restos dio lugar a que se tuviera que reestudiar la planta de vías, permitiendo:

- Garantizar la lectura global de los restos.

- Reducir al mínimo la afección de los restos, con el límite de garantizar una lectura de la totalidad.

- Garantizar la funcionalidad de las instalaciones ferroviarias en cualquier caso y dentro del marco del actual proyecto.

En la actualidad se están realizando, por un equipo técnico, el proyecto de compatibilización del edificio de viajeros con los restos citados, para permitir de esta forma adaptar el edificio inicialmente previsto a los requerimientos dados.

\section{SANTA JUSTA-SEVILLA}

Desde la década de los 60 se barajaba la solución de una estación única que liberara a la ciudad de los terrenos ocupados por las vías férreas, tanto en la zona del barrio de San Bernardo como en la calle Torneo, donde se ubicaban las dos estaciones simultáneas que ha tenido Sevilla desde el siglo pasado.

Santa Justa, desde el día 2 de mayo de 1991, es la nueva Estación de Viajeros de Sevilla, la quinta que se construye en la ciudad a lo largo de su historia. En ella coexisten todos los avances tecnológicos que, en materia de transporte ferroviario y tratamiento de edificios, encontramos a finales del siglo XX, dando lugar a las más avanzadas instalaciones que puedan encontrarse.

Su situación en la ciudad, así como los distintos accesos colindantes, le confieren una clara importancia centralizadora como nexo entre el núcleo urbano histórico y las nuevas zonas de expansión de la ciudad. 
Para describir someramente las instalaciones, podemos señalar que existen dos haces de vía: uno para Ancho Nacional de 6 vías vivas de viajeros y una de servicio, preparadas para pasar a ancho UIC; $y$ otro de 6 vías en Ancho Internacional (UIC), preparadas para enlazar con las vías de salida hacia Cádiz cuando se cambie esta línea.

Dispone de 2 vías de Autoexpreso en Ancho Internacional dentro del Edificio de Estación, y una en Ancho Nacional.

En edificios independientes se sitúan las naves de PaqueteExprés y Correos.

Para dar servicio a las vías de viajeros, existen 6 andenes de $525 \mathrm{~m}$ y 1 de $320 \mathrm{~m}$, con anchura en los centrales de 9 metros.

La cabeza de estación es el núcleo principal del edificio, ya que en él se encuentran situados la mayoría de los servicios, tanto al público como de uso interno ferroviario. Cabe distinguir el hall o vestíbulo principal (de $3.000 \mathrm{~m}^{2}$ ), perpendicular al haz de vías, situado a $6,25 \mathrm{~m}$ sobre el nivel de solería del Patio de andenes. En él se albergan: Taquillas, Información, Rail-Club, Cafeterias, Zonas Comerciales y, en general, servicios de atención al cliente.

En plantas superiores al hall se sitúan otros servicios, tales como: Centro de Control de Gestión, Oficinas y Zonas Comerciales futuras.

En total se alcanzan unos $5.500 \mathrm{~m}^{2}$ de zona comercial, $8.200 \mathrm{~m}^{2}$ de zona de uso público y $5.200 \mathrm{~m}^{2}$ de atención al cliente y dependencias.

Para acceder al edificio se ha construido una plaza, comunicada por ambos lados con la ciudad, que permite estructurar el tráfico viario para ofrecer una distribución adecuada de espacio destinada a taxis, tráfico privado y transporte colectivo. La superficie de esta zona es de unos $16.000 \mathrm{~m}^{2}$, circundada en el futuro por el Edificio de Oficinas que, con una superficie construida de $12.000 \mathrm{~m}^{2}$, permitirá albergar tanto servicios de RENFE como los que del exterior puedan ser necesarios.

Los aparcamientos se sitúan en ambos laterales del edificio, y uno pequeño en el frente de la estación para permanencias rápidas, con un total de 1.000 plazas.
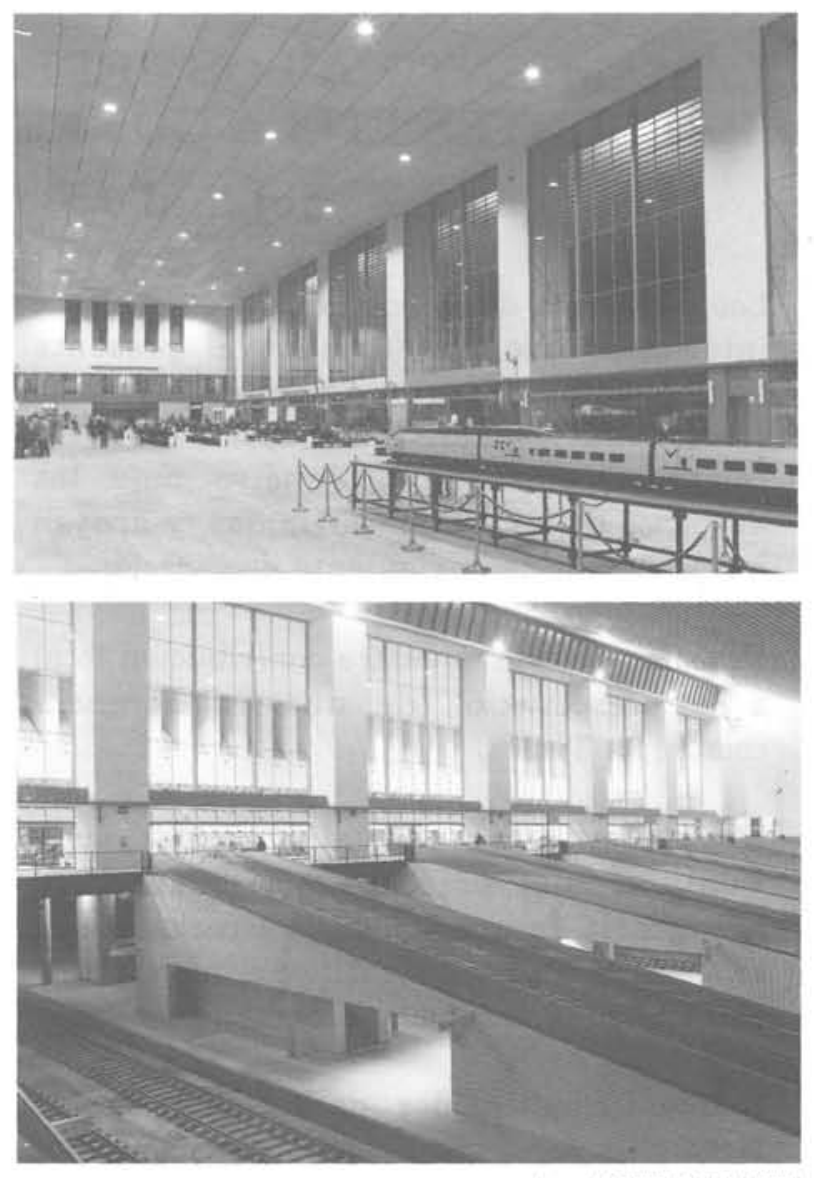

totos: MIGUEL ANGEL NISTAL

Interiores de la Estación de Santa Justa-Sevilla.

Asimismo, y rodeando estas zonas, se sitúa un viario que permite una circulación interior de vehículos.

La operación ferroviaria no se ha limitado a la citada estación, sino que se ha realizado una remodelación total de la Red Arterial Ferroviaria, permitiendo que se pueda cruzar, en el tren, la ciudad por túnel hacia Cádiz, dando una permeabilidad urbana inexistente anteriormente entre el casco antiguo y los distintos ensanches de la ciudad.

A la vez se han centrado los tráficos de mercancías, difuminados en toda la ciudad, a una estación central de mercancías en La Negrilla, y a un haz de vías de depósitos situados en el extrarradio de la ciudad, pero perfectamente comunicado con ella gracias a las distintas rondas urbanas construidas.

Todo ello ha dado lugar a la liberación de espacios para la ciudad, como la nueva calle Torneo, que ha permitido uno de los paseos más amplios y hermosos de Sevilla. 